\title{
351 - Success rate of various countermeasures against behavioral psychological symptoms of dementia based on the accumulation of real-world experience
}

Hiroaki Kazui, PhD; Shunsuke Sato; Kenji Yoshiyama; Hideki Kanemoto

Background and Objective: Appropriate countermeasures that can alleviate behavioral psychological symptoms of dementia (BPSD) are proposed. However, the effectiveness of these countermeasures has not been fully verified. Conversely, the caregivers of patients with dementia encounter BPSD every day and adopt some kind of measures against that particular BPSD.

Methods: We collected data regarding "whether or not a certain measure against a particular BPSD alleviates the symptom (care experience)" from all over Japan using Dementia Chienowa Net, which is a website we developed in 2016. We also collected patient's data, such as sex, primary disease, and nursing care level, which is an indicator of dementia severity in the Long-term Care Insurance System of Japan.

Results: In this study we analyzed 2003 care experiences (M/F:808/1192). In terms of primary disease, there were 1113 cases (55.6\%) of Alzheimer's dementia (AD), 236 cases (11.8\%) of dementia with Lewy bodies (DLB), 217 cases (10.8\%) of frontotemporal lobar degeneration (FTLD), and 118 cases (5.9\%) of vascular dementia $(\mathrm{VaD})$. Among $\mathrm{AD}$ patients living at home with nursing care level 1 , care experiences related to forgetfulness were the most common at $43 \%$, denial/rejection was $14 \%$, and restless behavior was $9 \%$. For nursing care level 2 , forgetfulness was $38 \%$, restless behavior was $15 \%$, and denial/rejection was $9 \%$. For nursing care level 3 , restless behavior was most common at $30 \%$, forgetfulness was $17 \%$, and denial/rejection was $16 \%$. Success rates were calculated; for "forgetting to take medicine," the success rate of "use of medicine box" was $40 \%$. The success rate of "use of medicine calendar" was $60.8 \%$, and the success rate of "someone handing over the medicine" was $92.3 \%$. For "poor fire management," the success rate of "changing to equipment that is less likely to cause hazards" was $71.4 \%$.

Conclusions: The frequency of DLB and FTLD in care experiences on Dementia Chienowa Net was more than the frequency of the diseases in Japan. The percentage of BPSD categories troubling patients' families differed depending on the severity of the dementia. The success rate of some common countermeasures against BPSD frequently encountered in daily life was embodied numerically. 